\title{
SCAMP4 enhances the senescent cell secretome
}

\author{
Kyoung Mi Kim, 1,3 Ji Heon Noh,,3 \\ Monica Bodogai, ${ }^{2}$ Jennifer L. Martindale, ${ }^{1}$ \\ Poonam R. Pandey, ${ }^{1}$ Xiaoling Yang, ${ }^{1}$ Arya Biragyn, ${ }^{2}$ \\ Kotb Abdelmohsen, ${ }^{1}$ and Myriam Gorospe ${ }^{1}$
}

${ }^{1}$ Laboratory of Genetics and Genomics, ${ }^{2}$ Laboratory of Molecular Biology and Immunology, National Institute on Aging Intramural Research Program, National Institutes of Health, Baltimore, Maryland 21224, USA

The senescence-associated secretory phenotype (SASP) is a major trait of senescent cells, but the molecular regulators of SASP factor secretion are poorly understood. Mass spectrometry analysis revealed that secretory carrier membrane protein 4 (SCAMP4) levels were strikingly elevated on the surface of senescent cells compared with proliferating cells. Interestingly, silencing SCAMP4 in senescent fibroblasts reduced the secretion of SASP factors, including interleukin 6 (IL6), IL8, growth differentiation factor 15 (GDF-15), C-X-C motif chemokine ligand 1 (CXCL1), and IL7, while, conversely, SCAMP4 overexpression in proliferating fibroblasts increased SASP factor secretion. Our results indicate that SCAMP4 accumulates on the surface of senescent cells, promotes SASP factor secretion, and critically enhances the SASP phenotype.

Supplemental material is available for this article.

Received February 16, 2018; revised version accepted May 22, 2018.

Cellular senescence is a state of indefinite growth arrest triggered in response to sublethal stresses such as telomere shortening, DNA damage, oxidative injury, oncogene activation, and hypoxia (Kuilman et al. 2010). Compared with proliferating cells, senescent cells have flattened and enlarged morphology, display heterochromatic DNA foci, and express distinct subsets of proteins, including the enzyme $\beta$-galactosidase (Goldstein et al. 1990; Dimri et al. 1995; Cristofalo et al. 1998). In addition, they display a major trait known as SASP (senescenceassociated secretory phenotype), encompassing a broad spectrum of secreted proteins such as proinflammatory cytokines, lymphokines, growth factors, angiogenic factors, and matrix metalloproteases that comprise the senescent cell secretome (Kuilman and Peeper 2009; Coppé et al. 2010). Through the SASP, senescent cells can change tissue function by promoting angiogenesis, recruiting immune cells, and remodeling the extracellular matrix (Acosta et al. 2013). In turn, these changes can alter organ function and accelerate the aging process.

[Keywords: human diploid fibroblasts; SCAMP4; senescence-associated secretory phenotype (SASP); cellular senescence]

${ }^{3}$ These authors contributed equally to this work. Corresponding author: kyoungmi.kim@nih.gov

Article is online at http://www.genesdev.org/cgi/doi/10.1101/gad.313270. 118 .
Cellular senescence is necessary in developmental morphogenesis, wound repair, the prevention of organ fibrosis, and tumor suppression in young individuals (Prieur and Peeper 2008; Storer et al. 2013; Demaria et al. 2014; Muñoz-Espín and Serrano 2014). However, it can also have detrimental effects as senescent cells accumulate in tissues with advancing age, contributing to age-related diseases such as cancer, cataracts, sarcopenia, and atherosclerosis (Ovadya and Krizhanovsky 2014; Sikora et al. 2014). Additionally, the age-associated accumulation of senescent cells has been linked to the heightened levels of SASP factors, which contributed to chronic inflammation and arthritis (Baker et al. 2008, 2011; Muñoz-Espín and Serrano 2014). SASP-associated proinflammatory cytokines and growth factors were further proposed to promote tumor growth by enhancing angiogenesis and tumor cell proliferation (Krtolica et al. 2001; Liu and Hornsby 2007; Coppé et al. 2008).

Supporting the notion that senescent cells exacerbate age-associated declines and diseases, the elimination of senescent cells in mice using genetic and pharmacological means led to improvements in age-related conditions such as cataracts, sarcopenia, loss of adipose tissue, and atherosclerosis and extended overall health span (Baker et al. 2011; Childs et al. 2017). A search for novel targets to detect and possibly intervene in senescence led us to identify dipeptidyl peptidase 4 (DPP4) as a membrane-associated protein highly expressed in senescent cells that enabled their selective elimination by natural killer cells (Kim et al. 2017). Here, we report the discovery of a plasma membrane-resident protein robustly up-regulated in senescent cells, SCAMP4 (secretory carrier membrane protein 4). We describe the mechanisms that suppress SCAMP4 production in proliferating cells and identify a critical function for SCAMP4 as a mediator of SASP factor secretion in senescent cells.

\section{Results and Discussion}

Identification of SCAMP4 as a novel cell surface protein highly enriched in senescent cells

We recently surveyed cell surface proteins differentially expressed in WI-38 human diploid fibroblasts that were either proliferating (at population doubling levels [PDLs] 20-25) or senescent following extended culture (PDLs 50-59); senescent cells displayed the characteristic flattened morphology and senescence-associated $\beta$-galactosidase (SA- $\beta$-gal) activity (Fig. $1 \mathrm{~A}_{\text {; }}$ Kim et al. 2017). In keeping with typical senescent traits, SASP factors interleukin 6 (IL6), IL8 (C-X-C motif chemokine ligand 8 [CXCL8]), IL1B (IL-1 $\beta$ ), growth differentiation factor 15 (GDF-15), and C-C motif chemokine ligand 2 (CCL2) were among those strongly up-regulated in senescent relative to proliferating populations (Fig. 1B). As reported (Kim et al. 2017), our survey identified several proteins differentially expressed on the plasma membrane of senescent compared with proliferating cells. Given that members of the SCAMP protein family had been implicated in cytokine secretion and exocytosis (Lin et al. 2005;

This is a work of the US Government. 
A

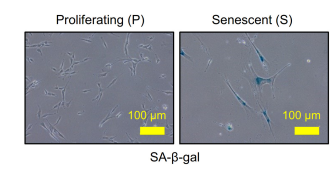

C

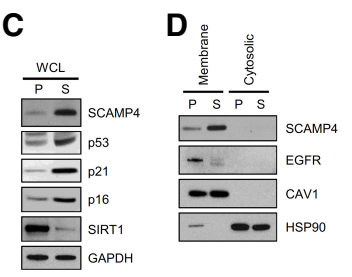

$\mathbf{E}$
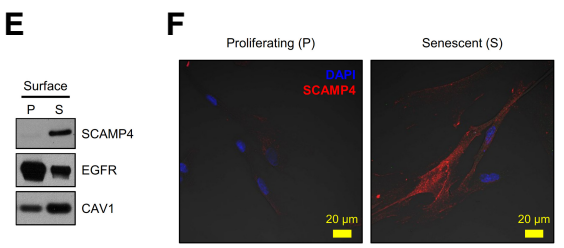

H

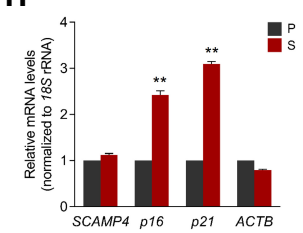

Figure 1. Identification of SCAMP4 as a cell surface protein selectively elevated in senescent WI-38 fibroblasts. (A) SA- $\beta$-gal analysis of proliferating (P; PDL 20) and senescent (S; PDL 50) WI-38 human diploid fibroblasts. (B) ELISA measurement of secreted IL6, IL8, GDF-15, CCL2, and IL1B in conditioned media from proliferating and senescent WI-38 cells, normalized to cell number. (ND) Not detectable. $(C-E)$ Western blot analysis of SCAMP4 levels in wholecell lysate (WCL) $(C)$, membrane and cytosolic lysates $(D)$, and cell surface $(E)$ from proliferating and senescent WI-38 fibroblasts. Senescence markers SIRT1, p53, p16, and p21; cytosolic marker HSP90; plasma membrane protein markers CAV1 (Caveolin-1) and EGFR; and loading control GAPDH were also assayed. $(F)$ Proliferating and senescent WI38 cells were fixed with $100 \%$ methanol, and endogenous SCAMP4 protein was detected by confocal microscopy. Merged signals. (Blue) Nuclei stained with DAPI; (red) SCAMP4. (G) Detached WI-38 (proliferating and senescent) cells were incubated with anti-SCAMP4-biotin or control human IgG-biotin antibodies and then incubated with streptavidin-APC antibodies. SCAMP4-positive cells were analyzed by flow cytometry; mean fluorescence intensity (MFI) of SCAMP4APC in proliferating and senescent cells was quantified. $(H)$ Steadystate SCAMP4 mRNA levels were measured by reverse transcription followed by quantitative PCR (RT-qPCR) analysis. p16 and p21 mRNAs were included as senescent cell markers, and ACTB mRNA was included as a loading control; mRNA levels were normalized to $18 \mathrm{~S}$ rRNA levels in each sample; mRNAs in proliferating cells were set as 1 . Graphs in $B, G$, and $H$ represent the means $\pm S E M$ from three independent experiments; $\left.\left.\right|^{* *}\right) P$-value $<0.01$.

Han et al. 2009), we sought to investigate in depth one of the proteins most strikingly up-regulated in senescent cells: SCAMP4.

We confirmed that SCAMP4 protein was selectively abundant in senescent WI-38 fibroblasts by Western blot analysis of proteins in whole-cell lysates, the membrane fraction, and the cell surface fraction (Fig. 1C-E). The lev- els of other SCAMP family proteins (SCAMP1-3) were also increased in senescent WI-38 fibroblasts, while SCAMP5 was not expressed in WI-38 fibroblasts (Supplemental Fig. S1). The localization of SCAMP4 on the plasma membrane of senescent WI-38 fibroblasts was further analyzed by immunofluorescence staining using methanol fixation without permeabilization to preserve the plasma membrane intact (Fig. 1F) and by flow cytometry analysis using a SCAMP4-biotin antibody, also without fixation or permeabilization (Fig. 1G; Materials and Methods). While these assays collectively confirmed that SCAMP4 increased on the plasma membrane outer surface of senescent cells, the levels of SCAMP4 mRNA, as measured by reverse transcription followed by quantitative PCR (RT-qPCR) analysis, did not change significantly in senescent cells relative to proliferating cells (Fig. 1H).

To test whether SCAMP4 levels increased similarly in other senescent models, we analyzed additional models of cell senescence. Ten days after exposing proliferating WI-38 and IMR-90 fibroblasts, human aortic endothelial cells (HAECs), and human umbilical vein endothelial cells (HUVECs) to ionizing radiation (IR), cells were rendered senescent, as confirmed by their positive SA- $\beta$-gal activity (Supplemental Fig. S2A-C). Western blot analyses revealed that SCAMP4 was up-regulated in each senescent population (Supplemental Fig. S2A-D). SCAMP4 also increased in doxorubicin (Doxo)-induced and oncogene-induced senescent WI-38 cells, as determined by Western blot analysis (Supplemental Fig. S2E,F). In each of these senescence models, SCAMP4 mRNA levels, as assessed by RT-qPCR analysis, did not change significantly (Supplemental Fig. S2).

Together, these data indicate that SCAMP4 protein is highly elevated on the surface of senescent cells without significant changes in SCAMP4 mRNA levels.

SCAMP4 is constitutively degraded in proliferating cells through the ubiquitin-proteasome system

To investigate how SCAMP4 protein levels increased in senescent cells compared with proliferating cells in the absence of changes in SCAMP4 mRNA levels, we first tested whether SCAMP4 protein was more actively translated in senescent fibroblasts. Polysomes present in lysates from proliferating (PDL 21) and senescent (PDL 54) fibroblasts were fractionated by centrifugation through linear $10 \%-50 \%$ sucrose gradients to separate ribosome subunits and monosomes (40S/60S/80S) from polysomes of low molecular weight (LMW) and high molecular weight (HMW) (Fig. 2A, left). RNA was isolated from each fraction and quantified by RT-qPCR analysis to determine the relative distribution of SCAMP4 mRNA and a control transcript, $A C T B$ mRNA, encoding the housekeeping protein ACTB ( $\beta$-Actin). As shown (Fig. $2 \mathrm{~A}$, right), SCAMP4 mRNA, like $A C T B$ mRNA, was distributed very similarly in proliferating and senescent cells and was largely found associated with polysomes, indicating that higher SCAMP4 translation did not explain the rise in SCAMP4 levels in senescent cells.

We thus examined instead whether SCAMP4 protein stability might differ between proliferating and senescent cells by measuring SCAMP4 protein half-life in WI-38 fibroblasts treated with the translation inhibitor cycloheximide (CHX). Blocking protein translation led to a rapid decline in SCAMP4 in proliferating cells, as determined 


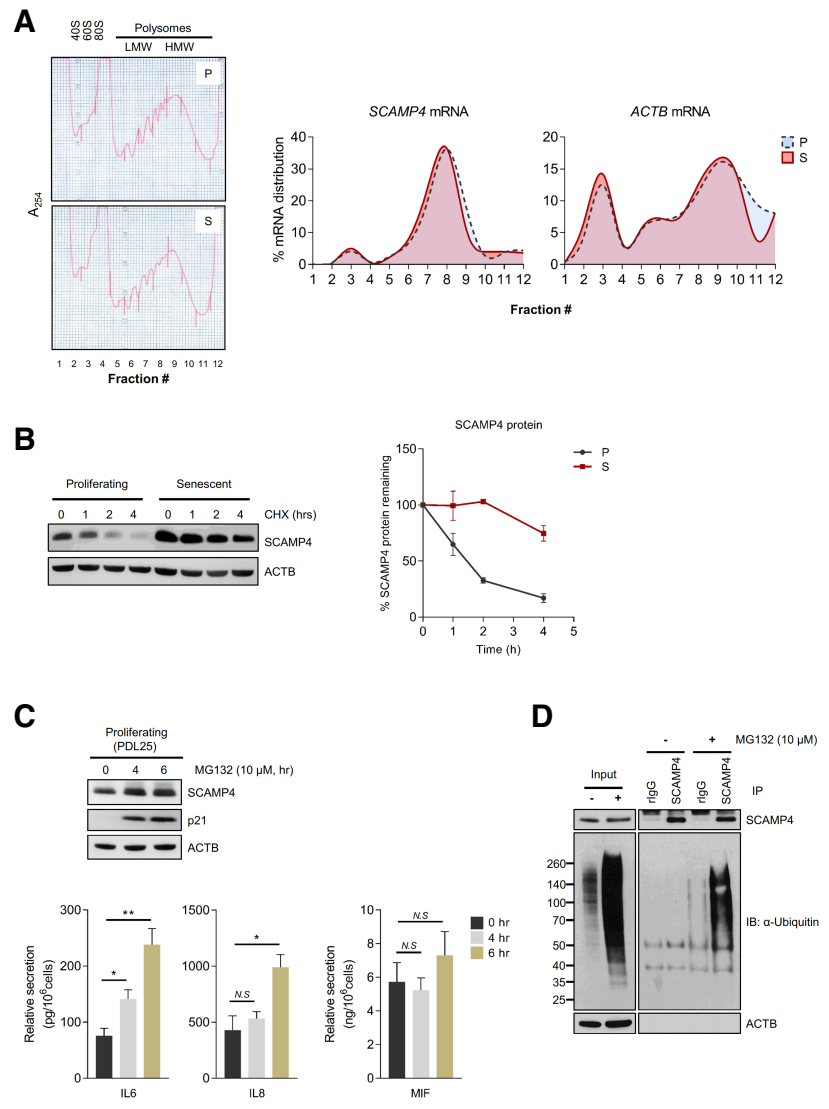

Figure 2. In proliferating cells, SCAMP4 is degraded by the ubiquitin-proteasome pathway. (A) Analysis of polysomes in proliferating (P) and senescent (S) cells. Lysates of proliferating and senescent WI-38 cells were loaded onto $10 \%-50 \%$ linear sucrose gradients, and the relative distributions of SCAMP4 mRNA and ACTB (control) mRNA were calculated after RT-qPCR analysis in each fraction. $(B)$ The stability of the SCAMP4 protein in proliferating and senescent cells. (Left) Western blot analysis of the levels of SCAMP4 and loading control ACTB in cells harvested after treatment with the protein synthesis inhibitor cycloheximide (CHX) for the times shown. (Right) Quantification of SCAMP4 signals normalized to ACTB signals. Data are the means \pm SEM from two independent experiments. (C) Proliferating WI-38 fibroblasts (PDLs 22-25) were incubated with either vehicle (ethanol) or $10 \mu \mathrm{M}$ MG132 for 4 or $6 \mathrm{~h}$. (Top) Western blot analysis of SCAMP4 levels in WI-38 fibroblasts treated with MG132 for 4 or $6 \mathrm{~h}$. (p21) Marker of proteasome inhibition; (ACTB) loading control. (Bottom) ELISA measurement of secreted IL6, IL8, and MIF. ( $D$ ) Immunoprecipitation using anti-SCAMP4 or rIgG (control) antibodies in proliferating cells with or without MG132 for 3 h. After lysis, ubiquitinated SCAMP4 was detected by Western blot analysis using anti-ubiquitin antibody. ACTB was detected in lysates (Input) used in immunoprecipitation reactions. $\left(^{* *}\right) P$-value $<0.01 ;\left(^{*}\right)$ $P$-value $<0.05$; (N.S.) not significant.

by Western blot analysis (with an apparent half-life of $\sim 1.5 \mathrm{~h}$ ), while SCAMP4 was quite stable in senescent cells; the control protein ACTB was stable in both populations (Fig. 2B). To distinguish between two major pathways of protein stability (the ubiquitin-proteasome degradation system [UPS] and autophagy), proliferating and senescent cells were treated with the proteasome inhibitor MG132 or the autophagy inhibitor bafilomycin A1. Cells were harvested $24 \mathrm{~h}$ later, and SCAMP4 protein was found to accumulate when the proteasome was inhibited in proliferating cells but not in senescent cells, as de- termined by Western blot analysis (Supplemental Fig. S3A). The levels of senescence markers and secreted SASP factors increased after MG132 treatment (Supplemental Fig. S3A,B). To address whether SCAMP4 might be stabilized when the proteasome was inhibited but before cells reached senescence, we measured SCAMP4 levels after short-term treatment with MG132. Western blot analysis revealed that SCAMP4 accumulated after MG132 treatment by $4 \mathrm{~h}$, before SASP factor secretion was widely induced (Fig. 2C); there was no increase by 2 $\mathrm{h}$ (data not shown). In contrast, SCAMP4 accumulated in both proliferating and senescent cells after inhibiting autophagy (Supplemental Fig. S3C), indicating that UPS, but not autophagy, was responsible for the selective reduction of SCAMP4 in proliferating cells.

In keeping with the finding that SCAMP4 was ubiquitinated on Lys4 and Lys185 in lung cancer cells (Wu et al. 2015), we found that SCAMP4 was ubiquitinated in MG132-treated proliferating WI-38 cells, as determined by SCAMP4 immunoprecipitation followed by Western blot analysis of ubiquitinated (Ub) proteins (Fig. 2D). These results indicate that SCAMP4 protein was selectively unstable and degraded by ubiquitin-mediated proteolysis in proliferating cells, while, in senescent cells, SCAMP4 was stable and accumulated to high levels.

\section{Silencing SCAMP4 reduces the production and secretion of SASP factors}

Given earlier evidence that SCAMP5, a member of the SCAMP protein family, promoted cytokine secretion in human epithelial cancer cells and human monocytes (Han et al. 2009), we tested whether SCAMP4 might affect senescence traits, particularly SASP. SCAMP4 was silenced in presenescent fibroblasts by transfecting siRNAs; $72 \mathrm{~h}$ later, RT-qPCR analysis revealed moderate reductions in the levels of mRNAs encoding some well-known SASP factors (IL1A and IL1B mRNAs, encoding IL1A [IL-1 $\alpha$ ] and IL1B [IL-1 $\beta$ ], respectively) but no changes for IL6 or IL8 mRNAs (Fig. 3A). Importantly, senescence markers SA- $\beta$-gal activity, p53 and p 1 protein levels (Fig. 3B,C), and the levels of SASP factor IL1B all decreased in SCAMP4 silenced WI-38 cells. Considering that IL1A can regulate SASP secretion (Orjalo et al. 2009) and that SCAMP5 affects CCL5 secretion (Han et al. 2009), we sought to study comprehensively whether SCAMP4 might regulate cytokine secretion. Interestingly, analysis of conditioned medium from fibroblasts using a cytokine array revealed that the secretion of many cytokines decreased after silencing SCAMP4 (Fig. 3D). To validate these results, we measured the levels of several major SASP factors (IL6, IL8, GDF-15, IL1B, and CCL2) secreted into the culture medium using ELISA; indeed, by $72 \mathrm{~h}$ after silencing SCAMP4, several cytokines were markedly reduced in the culture medium of presenescent cells, including IL8, MIF, and IL1B (Supplemental Fig. S3D).

To test whether SCAMP4 affects SASP secretion in senescent cells, we triggered WI-38 senescence by exposure to IR, subsequently silenced SCAMP4, and measured the secretion of SASP factors into the culture medium by ELISA (Fig. 3E). As shown in Figure 3F, the secretion of several SASP factors by senescent cells declined if SCAMP4 was silenced, further supporting a role for SCAMP4 in enhancing SASP factor secretion in senescent cells. 

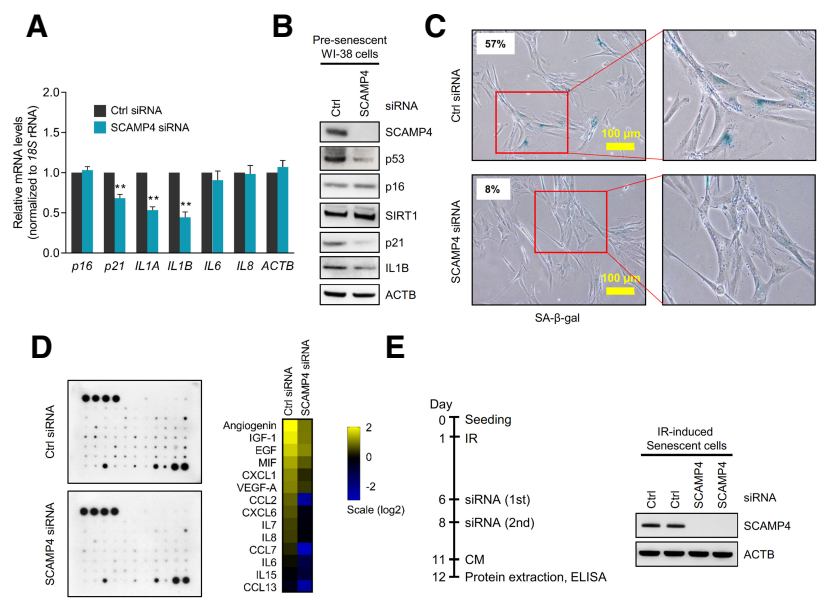

E

F

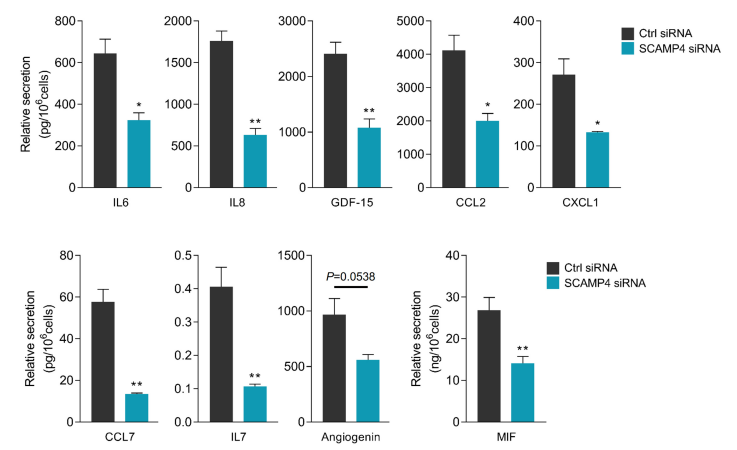

Figure 3. Silencing SCAMP4 in WI-38 fibroblasts reduces the secretion of SASP factors. $(A-D)$ Seventy-two hours after transfection with control (Ctrl) siRNA or SCAMP4 siRNA, presenescent WI-38 cells (PDLs 39-42) were analyzed. (A) Levels of p16, p21, IL1A, IL1B, IL6, $I L 8$, and $A C T B$ mRNAs, as measured by RT-qPCR analysis and normalized to $18 \mathrm{~S}$ rRNA levels. $(B)$ Western blot analysis to assess the levels of SCAMP4, p53, p16, SIRT1, p21, IL1B, and ACTB in wholecell lysates. (C) SA- $\beta$-gal-stained cells (the percentage of $\beta$-gal positive cells is shown). (D) Cytokine array analysis of the secreted factors $(l e f t)$ and array signal quantification (right). (E) Experimental scheme (left) and Western blot analysis of SCAMP4 levels (right). (F) ELISA to quantify the levels of secreted IL6, IL8, GDF-15, CCL2, CXCL1, CCL7, IL7, angiogenin, and MIF. Graphs in $A$ and $F$ represent the means \pm SEM from three independent experiments. $\left(^{* *}\right) P$-value $<$ $0.01 ;\left(^{*}\right) P$-value $<0.05$.

\section{SCAMP4 overexpression promotes the secretion of SASP factors}

Given that secretion of SASP factors was reduced in SCAMP4-depleted cells, we asked whether, conversely, elevating SCAMP4 might promote the secretion of SASP factors. To test this possibility, we overexpressed SCAMP4 in proliferating WI-38 cells (PDL 25) by infection with a lentivirus that expressed SCAMP4-Myc; control viral particles expressed only the Myc tag. After selecting the infected cells in $1 \mu \mathrm{g} / \mathrm{mL}$ puromycin for $20 \mathrm{~d}$, RTqPCR analysis revealed that the levels of senescent markers p16 mRNA and mRNAs encoding SASP factors IL1B, IL6, and IL8 were higher in cells overexpressing SCAMP4 (Fig. 4A). SA- $\beta$-gal signals also increased after overexpressing SCAMP4 (Fig. 4B), and Western blot analysis similarly confirmed that the levels of senescent markers p53 and p16 as well as SASP factors IL1A and IL1B increased after expressing SCAMP4-Myc (Fig. 4C). In addition, IL1A
mRNA was more actively translated in SCAMP4-Myc-expressing fibroblasts, as determined by analyzing its association with heavy polysomes (Supplemental Fig. S4A). SCAMP4 overexpression also affected cell proliferation, as revealed by the reduced incorporation of $\left[{ }^{3} \mathrm{H}\right]$-thymidine in cells overexpressing SCAMP4 relative to control cells (Fig. 4D). In addition, the levels of IL6, IL8, GDF15, IL1B, CXCL1, and IL7 secreted in the medium increased in SCAMP4-overexpressing cells compared with control cells (Fig. 4E).

Short-term SCAMP4 overexpression similarly affected SASP secretion and senescence. By $3 \mathrm{~d}$ of SCAMP4-Myc overexpression in proliferating WI-38 cells (Supplemental Fig. S4B), RT-qPCR analysis revealed increased levels of the mRNAs encoding p16 and SASP factors GDF-15, MIF, IL1A, IL1B, IL6, IL8, and CXCL1 (Supplemental Fig. S4C), while ELISA indicated higher secretion of IL6,

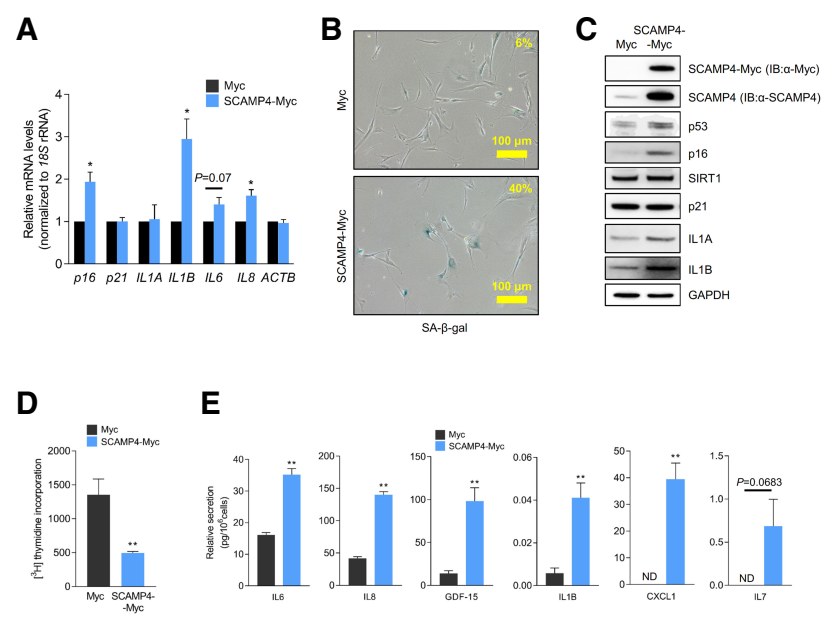

$\mathbf{F}$

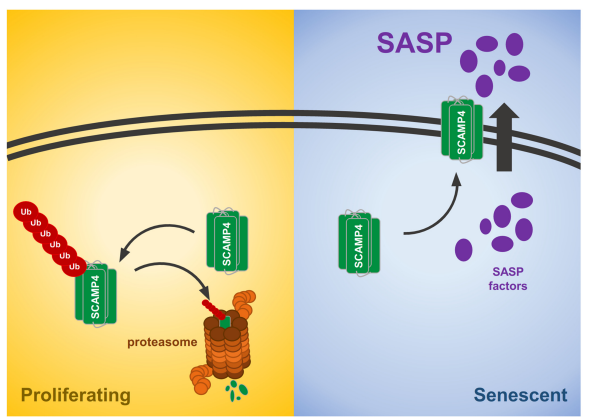

Figure 4. SCAMP4 promotes secretion of SASP factors. $(A-E)$ Proliferating WI-38 cells were transduced with lentiviral particles to express either Myc tag alone or SCAMP4-Myc. Twenty days later, RT-qPCR analysis was used to measure the steady-state levels of p16, p21, IL1A,IL1B,IL6,IL8, and ACTB mRNAs (normalized to $18 S$ rRNA levels) $(A)$; SA- $\beta$-gal activity was assessed $(B)$; Western blot analysis was performed to assess the levels of SCAMP4-Myc (using anti-SCAMP4 antibody or anti-Myc antibody), p53, IL1A, IL1B, p21, p16, SIRT1, and GAPDH (loading control) in whole-cell lysates $(C) ;\left[{ }^{3} \mathrm{H}\right]$-thymidine incorporation was measured $(D)$; and the secretion of IL6, IL8, GDF-15, IL1B, CXCL1, and IL7 was measured by ELISA $(E)$. Graphs in $A, D$, and $E$ represent the means \pm SEM from three independent experiments. $\left(^{* *}\right) P$-value $<0.01 ;\left(^{*}\right) P$-value $<0.05$. $(F)$ Proposed model. In proliferating cells, SCAMP4 is rapidly degraded by the ubiquitin-proteasome degradation system. In senescent cells, SCAMP4 is stable and localizes to the cell surface, in turn promoting the secretion of SASP factors during senescence. 
IL8, GDF-15, CXCL1, and MIF in SCAMP4-overexpressing cells (Supplemental Fig. S4D).

In summary, our data indicate that SCAMP4, a cell surface protein highly up-regulated in senescent cells, can promote the secretion of SASP factors. Accordingly, we suggest a model in which SCAMP4 protein is rapidly degraded by the ubiquitin-proteasome pathway in proliferating cells, while it is stable in senescent cells, accumulates, localizes on the cell surface, and stimulates SASP factor secretion. By promoting the secretion of SASP factors, we propose that SCAMP4 enables one of the major phenotypes of cellular senescence and further enhances local cell senescence (Fig. 4F), given the autocrine actions of several SASP factors (Lunyak et al. 2017).

\section{Implications of SCAMP4 on senescence and aging}

The impact of senescent cells in physiology (e.g., wound repair and morphogenesis) and pathology (e.g., cancer, arthritis, cataracts, and atherosclerosis) has been attributed to factors present in the SASP, the senescent cell secretome. Several levels of regulation of the senescent secretome have been reported. Upstream regulatory events include activation of signaling cascades by factors including the prominent regulator IRAK-1 and downstream mitogen-activated protein kinases p38 and JNK. These inducers activate prominent transcriptional regulators of SASP factor production in senescent cells, notably NF- $\kappa B$ and C/EBP $\beta$, which induce the transcription of SASP genes such as IL6 and IL8 (Orjalo et al. 2009). At the post-transcriptional level, numerous regulators of the stability and translation of mRNAs encoding SASP factors have also been identified, including several RNA-binding proteins (e.g., ILF3/NF-90), microRNAs (e.g., miR-146a/b, miR-335, miR-15b, and miR-34a), long noncoding RNAs (e.g., THRIL, NEAT1, Lethe, etc), and circular RNAs (e.g., CircPVT1 and Circ-Foxo3) (Tominaga-Yamanaka et al. 2012; Steri et al. 2017; for review, see Panda et al. 2017). However, the mechanisms by which cytokines are secreted by senescent cells are not well understood, although there is evidence that they include exocytosis, direct protein secretion, and secretion via vesicles such as exosomes.

In this regard, among the ubiquitous SCAMP family members (Fernández-Chacón and Südhof 2000; Han et al. 2009), SCAMP1, SCAMP2, and SCAMP3 have N-terminal cytoplasmic NPF (Arg-Pro-Phe) repeats, transmembrane regions (TMRs), and a cytoplasmic tail involved in endocytosis through trafficking Golgi to the plasma membrane (Castle and Castle 2005). However, SCAMP4 and SCAMP5 lack the cytoplasmic NPF repeats and localize on plasma membranes. SCAMP5 was found to promote the secretion of CCL5, a cytokine containing a signal peptide regulated by calcium, in response to signals that promote calcium influx in human epithelial and immune cells (Han et al. 2009). SCAMP5 regulated the secretion of CCL5 via interaction with SNARE (soluble N-ethylmaleimide-sensitive factor attachment protein receptors) proteins. Whether SCAMP4 also functions in the secretion of specific cytokines through the SNARE machinery (Stanley and Lacy 2010), facilitates exosome release, or promotes direct secretion of targets such as IL1B, MIF, and IL8 is unknown. Studies are under way to identify SCAMP4-interacting proteins in order to uncover possible mediators of its secretory function.
In light of the profound impact of senescent cells upon organ physiology and disease and the prominence of the senescent cell secretome, the finding that SCAMP4 induces SASP factor secretion (Figs. 3, 4) underscores the potential value of SCAMP4 as a target of therapeutic intervention. Enhancing SCAMP4 function thus might be advantageous in situations in which senescent cells are beneficial, such as wound healing and fibrosis prevention. Conversely, suppressing SCAMP4 function may thwart the detrimental actions of senescent cells in many age-associated conditions, including chronic inflammation, arthritis, sarcopenia, diabetes, and cancer.

\section{Materials and methods}

Cell culture, establishment of senescence, transfection, and $S A-\beta$-gal activity

Human diploid fibroblasts WI-38 (Coriell Cell Repositories) and other senescence models were cultured as described in the Supplemental Material. Proliferating WI-38 human diploid fibroblasts were used at PDLs ranging between PDL 20 and 26, and senescent WI-38 human diploid fibroblasts were used after additional culture (PDLs 50-59). Gene silencing and overexpression analyses are described in the Supplemental Material. Control (UUCUCCGAACGUGUCACGU) (IDT) and SCAMP4 (ACAGAACCCG UGUUCAUCU) (Thermo Scientific) siRNAs were transfected at $50 \mathrm{nM}$ using Lipofectamine 2000 (Thermo Scientific). Lentiviruses were used to overexpress either SCAMP4-Myc or Myc (GeneCopoeia, Inc.). Thymidine incorporation and flow cytometric analyses are described (Supplemental Material).

\section{Protein analysis}

Proteins on the cell membrane, on the cell surface, and in whole-cell lysates were analyzed by Western blot analysis using primary antibodies that were detected by secondary antibodies and visualized using enhanced chemiluminescence (Supplemental Material; Noh et al. 2016). Protein stability was assessed by incubating WI-38 cells with $100 \mu \mathrm{g} / \mathrm{mL}$ CHX (Calbiochem). Following immunoprecipitation of WI-38 cell lysates, the presence of bound proteins was assessed by Western blot analysis as described (Supplemental Material).

\section{Analysis of secreted proteins: cytokine arrays and ELISA}

Conditioned medium (CM) was prepared by washing cells twice with $1 \times$ PBS, adding fresh DMEM with $0.2 \%$ FBS, incubating cells for an additional 24-36 h, and collecting the CM for further analysis. Individual cytokines secreted into the culture medium were measured using either human Quantikine ELISA kits (R\&D Systems) or cytokine arrays (RayBiotech) (Supplemental Material).

\section{RNA isolation and RT- $q P C R$ analysis}

Total RNA was extracted using TriPure isolation reagent (Roche). RT-qPCR analysis was used to measure the levels of specific mRNAs using gene-specific primer pairs (Supplemental Material), SYBR Green master mix (Kapa Biosystems), and an Applied Biosystems 7300 instrument. Polysome fractionation and analysis of polysome-associated RNA were performed as described in the Supplemental Material and Tominaga-Yamanaka et al. (2012).

Immunofluorescence microscopy

Confocal microscopy was performed as described (Supplemental Material; Kim et al. 2017).

\section{Acknowledgments}

We thank F.E. Indig for help with confocal imaging. This work was funded in its entirety by the National Institute on Aging Intramural Research Program, National Institutes of Health. 
Author contributions: K.M.K., J.H.N., M.B., J.L.M., P.R.P., K.A., A.B., and M.G. performed experiments and analyzed data. X.Y. prepared the reagents. K.M.K., J.H.N., and M.G. interpreted the results and wrote the paper.

\section{References}

Acosta JC, Banito A, Wuestefeld T, Georgilis A, Janich P, Morton JP, Athineos D, Kang TW, Lasitschka F, Andrulis M, et al. 2013. A complex secretory program orchestrated by the inflammasome controls paracrine senescence. Nat Cell Biol 15: 978-990.

Baker DJ, Perez-Terzic C, Jin F, Pitel KS, Niederländer NJ, Jeganathan K, Yamada S, Reyes S, Rowe L, Hiddinga HJ, et al. 2008. Opposing roles for p16Ink4a and p19Arf in senescence and ageing caused by BubR1 insufficiency. Nat Cell Biol 10: 825-836.

Baker DJ, Wijshake T, Tchkonia T, LeBrasseur NK, Childs BG, van de Sluis B, Kirkland JL, van Deursen JM. 2011. Clearance of p16Ink4a-positive senescent cells delays ageing-associated disorders. Nature 479: 232-236.

Castle A, Castle D. 2005. Ubiquitously expressed secretory carrier membrane proteins (SCAMPs) 1-4 mark different pathways and exhibit limited constitutive trafficking to and from the cell surface. J Cell Sci 118: 3769-3780.

Childs BG, Gluscevic M, Baker DI, Laberge RM, Marquess D, Dananberg J, van Deursen JM. 2017. Senescent cells: an emerging target for diseases of ageing. Nat Rev Drug Discov 16: 718-735

Coppé JP, Patil CK, Rodier F, Sun Y, Muñoz DP, Goldstein J, Nelson PS, Desprez PY, Campisi J. 2008. Senescence-associated secretory phenotypes reveal cell-nonautonomous functions of oncogenic RAS and the p53 tumor suppressor. PLoS Biol 6: 2853-2868.

Coppé JP, Desprez PY, Krtolica A, Campisi J. 2010. The senescence-associated secretory phenotype: the dark side of tumor suppression. Annu Rev Pathol 5: 99-118.

Cristofalo VI, Volker C, Francis MK, Tresini M. 1998. Age-dependent modifications of gene expression in human fibroblasts. Crit Rev Eukaryot Gene Expr 8: 43-80.

Demaria M, Ohtani N, Youssef SA, Rodier F, Toussaint W, Mitchell JR, Laberge RM, Vijg J, Van Steeg H, Dollé ME, et al. 2014. An essential role for senescent cells in optimal wound healing through secretion of PDGF-AA. Dev Cell 31: 722-733.

Dimri GP, Lee X, Basile G, Acosta M, Scott G, Roskelley C, Medrano EE, Linskens M, Rubelj I, Pereira-Smith O, et al. 1995. A biomarker that identifies senescent human cells in culture and in aging skin in vivo. Proc Natl Acad Sci 92: 9363-9367.

Fernández-Chacón R, Südhof TC. 2000. Novel SCAMPs lacking NPF repeats: ubiquitous and synaptic vesicle-specific forms implicate SCAMPs in multiple membrane-trafficking functions. J Neurosci 20: 7941-7950.

Goldstein S. 1990. Replicative senescence: the human fibroblast comes of age. Science 249: 1129-1133.

Han C, Chen T, Yang M, Li N, Liu H, Cao X. 2009. Human SCAMP5, a novel secretory carrier membrane protein, facilitates calcium-triggered cytokine secretion by interaction with SNARE machinery. I Immunol 182: 2986-2996.
Kim KM, Noh JH, Bodogai M, Martindale JL, Yang X, Indig FE, Basu SK, Ohnuma K, Morimoto C, Johnson PF, et al. 2017. Identification of senescent cell surface targetable protein DPP4. Genes Dev 31: 1529-1534.

Krtolica A, Parrinello S, Lockett S, Desprez PY, Campisi J. 2001. Senescent fibroblasts promote epithelial cell growth and tumorigenesis: a link between cancer and aging. Proc Natl Acad Sci 98: 12072-12077.

Kuilman T, Peeper DS. 2009. Senescence-messaging secretome: SMS-ing cellular stress. Nat Rev Cancer 9: 81-94.

Kuilman T, Michaloglou C, Mooi WJ, Peeper DS. 2010. The essence of senescence. Genes Dev 24: 2463-2479.

Lin PJ, Williams WP, Luu Y, Molday RS, Orlowski J, Numata M. 2005 Secretory carrier membrane proteins interact and regulate trafficking of the organellar $\left(\mathrm{Na}^{+}, \mathrm{K}^{+}\right) / \mathrm{H}^{+}$exchanger NHE7. I Cell Sci 118: 1885-1897.

Liu D, Hornsby PJ. 2007. Senescent human fibroblasts increase the early growth of xenograft tumors via matrix metalloproteinase secretion. Cancer Res 67: 3117-3126.

Lunyak VV, Amaro-Ortiz A, Gaur M. 2017. Mesenchymal stem cells secretory responses: senescence messaging secretome and immunomodulation perspective. Front Genet 8: 220.

Muñoz-Espín D, Serrano M. 2014. Cellular senescence: from physiology to pathology. Nat Rev Mol Cell Biol 15: 482-496.

Noh JH, Kim KM, Abdelmohsen K, Yoon JH, Panda AC, Munk R, Kim J, Curtis J, Moad CA, Wohler CM, et al. 2016. HuR and GRSF1 modulate the nuclear export and mitochondrial localization of the lncRNA RMRP. Genes Dev 30: 1224-1239.

Orjalo AV, Bhaumik D, Gengler K, Scott GK, Campisi J. 2009. Cell surfacebound IL-1 $\alpha$ is an upstream regulator of the senescence-associated IL6/IL-8 cytokine network. Proc Natl Acad Sci 106: 17031-17036.

Ovadya Y, Krizhanovsky V. 2014. Senescent cells: SASPected drivers of age-related pathologies. Biogerontology 15: 627-642.

Panda AC, Abdelmohsen K, Gorospe M. 2017. SASP regulation by noncoding RNA. Mech Ageing Dev 168: 37-43.

Prieur A, Peeper DS. 2008. Cellular senescence in vivo: a barrier to tumorigenesis. Curr Opin Cell Biol 20: 150-155.

Sikora E, Bielak-Zmijewska A, Mosieniak G. 2014. Cellular senescence in ageing, age-related disease and longevity. Curr Vasc Pharmacol 12: 698-706.

Stanley AC, Lacy P. 2010. Pathways for cytokine secretion. Physiology 25: 218-229.

Steri M, Orrù V, Idda ML, Pitzalis M, Pala M, Zara I, Sidore C, Faà V, Floris M, Deiana M, et al. 2017. Overexpression of the Cytokine BAFF and Autoimmunity Risk. N Eng1 J Med 376: 1615-1626.

Storer M, Mas A, Robert-Moreno A, Pecoraro M, Ortells MC, Di Giacomo V, Yosef R, Pilpel N, Krizhanovsky V, Sharpe J, et al. 2013. Senescence is a developmental mechanism that contributes to embryonic growth and patterning. Cell 155: 1119-1130.

Tominaga-Yamanaka K, Abdelmohsen K, Martindale JL, Yang X, Taub DD, Gorospe M. 2012. NF90 coordinately represses the senescence-associated secretory phenotype. Aging 4: 695-708.

Wu Q, Cheng Z, Zhu J, Xu W, Peng X, Chen C, Li W, Wang F, Cao L, Yi X, et al. 2015. Suberoylanilide hydroxamic acid treatment reveals crosstalks among proteome, ubiquitylome and acetylome in non-small cell lung cancer A549 cell line. Sci Rep 5: 9520. 


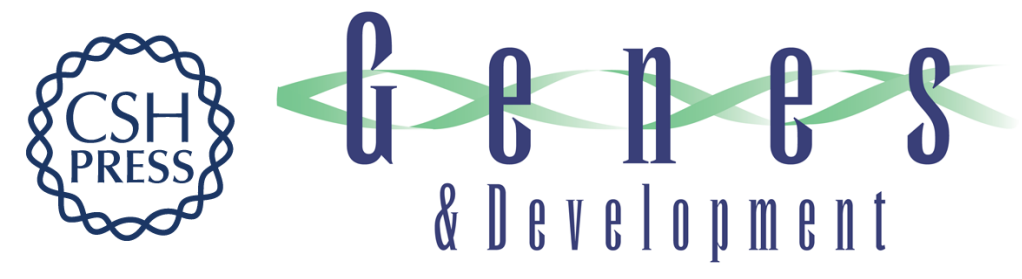

\section{SCAMP4 enhances the senescent cell secretome}

Kyoung Mi Kim, Ji Heon Noh, Monica Bodogai, et al.

Genes Dev. 2018, 32:

Access the most recent version at doi:10.1101/gad.313270.118

\section{Supplemental http://genesdev.cshlp.org/content/suppl/2018/07/02/32.13-14.909.DC1 \\ Material}

References This article cites 32 articles, 12 of which can be accessed free at: http://genesdev.cshlp.org/content/32/13-14/909.full.html\#ref-list-1

License This is a work of the US Government.

Email Alerting Receive free email alerts when new articles cite this article - sign up in the box at the top Service right corner of the article or click here.

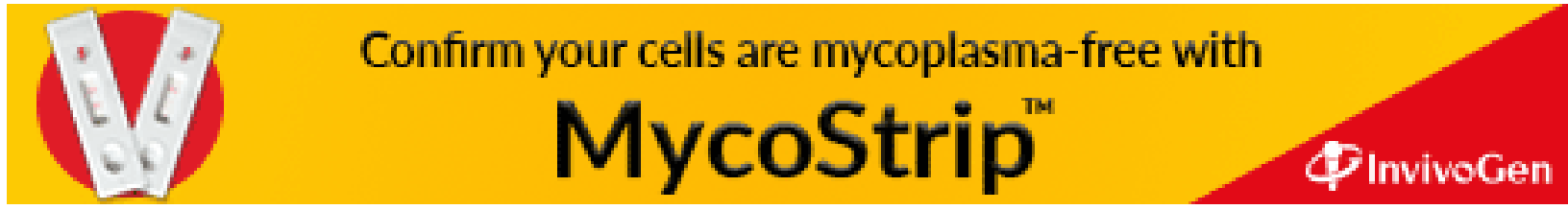

